\title{
POTENTIALLY STABLE AND 5-BY-5 SPECTRALLY ARBITRARY TREE SIGN PATTERN MATRICES WITH ALL EDGES NEGATIVE*
}

\author{
SUNIL DAS ${ }^{\dagger}$
}

\begin{abstract}
Characterization of potentially stable sign pattern matrices has been a long-standing open problem. In this paper, we give some sufficient conditions for tree sign pattern matrices with all edges negative to allow a properly signed nest. We also characterize potentially stable star and path sign pattern matrices with all edges negative. We give a conjecture on characterizing potentially stable tree sign pattern matrices with all edges negative in terms of allowing a properly signed nest which is verified to be true for sign pattern matrices up to order 6 . Finally, we characterize all 5-by-5 spectrally arbitrary tree sign pattern matrices with all edges negative.
\end{abstract}

Key words. Potentially stable, Spectrally arbitrary, Tree, Sign pattern, Properly signed nest.

AMS subject classifications. 15A18, 15B35, 05C50.

1. Introduction. A sign pattern matrix is a matrix with entries from $\{+,-, 0\}$. If $M_{m \times n}(\mathbb{R})$ is the set of all $m \times n$ real matrices, then the qualitative class of an $m \times n$ sign pattern matrix $A$ is denoted by $Q(A)$ and is defined by:

$$
Q(A)=\left\{C \in M_{m \times n}(\mathbb{R}): \operatorname{sign}\left(c_{i j}\right)=a_{i j} \text { for all } i \in\{1,2, \ldots, m\}, j \in\{1,2, \ldots, n\}\right\} .
$$

A sign pattern matrix $A$ allows a property $P$ if at least one matrix in $Q(A)$ has the property $P$ and requires a property $P$ if all matrices in $Q(A)$ have the property $P$. A square sign pattern matrix $A=\left[a_{i j}\right]$ is said to be combinatorially symmetric if for each $i, j$, either both $a_{i j}, a_{j i}$ are zero or both $a_{i j}, a_{j i}$ are nonzero. Let us recall some definitions from [12].

A subpattern $\tilde{A}$ of a sign pattern matrix $A$ is a sign pattern matrix obtained from $A$ by replacing some (possibly none) of the nonzero entries of $A$ with 0 . In this case, $A$ is said to be a super-pattern of $\tilde{A}$.

A diagonal sign pattern matrix is a square sign pattern matrix with all off-diagonal entries 0 . A signature sign pattern matrix is a diagonal sign pattern matrix with each diagonal entry + or - . A signature similarity of a square sign pattern matrix $A$ is a product $S A S$, where $S$ is a signature sign pattern matrix.

A permutation sign pattern matrix is a square sign pattern matrix with entries from $\{0,+\}$, where the entry + occurs precisely once in each row and each column. A permutation similarity of a square sign pattern matrix $A$ is a product $P^{T} A P$, where $P$ is a permutation sign pattern matrix.

A sign pattern matrix $B$ is equivalent to another sign pattern matrix $A$ if $B$ is obtained from $A$ by negation and/or permutation similarity and/or signature similarity.

For a simple undirected graph $G$, we denote the edge between two vertices $i, j$ by $[i, j]$. Let us recall from [13] that the graph of a matrix $A$ of order $n$, denoted by $G(A)$, is defined to be a simple undirected graph with vertices $1,2, \ldots, n$ and for $i \neq j$ it has the edge $[i, j]$ if and only if $a_{i j} \neq 0$ or $a_{j i} \neq 0$. A sign

\footnotetext{
* Received by the editors on March 11, 2021. Accepted for publication on July 9, 2021. Handling Editor: Michael Tsatsomeros.

${ }^{\dagger}$ Department of Mathematics, Indian Institute of Technology Guwahati, Assam-781039, India (sunil.das@iitg.ac.in).
} 
563 Potentially stable and 5-by-5 spectrally arbitrary tree sign pattern matrices with all edges negative

pattern matrix $A$ is a tree sign pattern matrix if $A$ is combinatorially symmetric and $G(A)$ is a tree. In particular, if $G(A)$ is a star or a path, then $A$ is called a star sign pattern matrix or a path sign pattern matrix, respectively.

The eigenvalues of matrices in the qualitative class of a tree sign pattern matrix $A$ depend on the signs $a_{i i}$ and $a_{i j} a_{j i}$. Therefore each tree sign pattern matrix can be identified by a signed tree whose vertices may be signed,,+- 0 and whose edges may be signed,+- . Vertex $i$ has the $\operatorname{sign} a_{i i}$, and for $i \neq j$, if $a_{i j} a_{j i} \neq 0$, then the edge between vertices $i$ and $j$ has the sign $a_{i j} a_{j i}$. For example, a sign pattern matrix with its signed tree is as follows:

$$
\left[\begin{array}{ccccc}
0 & - & 0 & + & 0 \\
- & 0 & + & 0 & - \\
0 & - & + & 0 & 0 \\
- & 0 & 0 & + & 0 \\
0 & - & 0 & 0 & 0
\end{array}\right]
$$

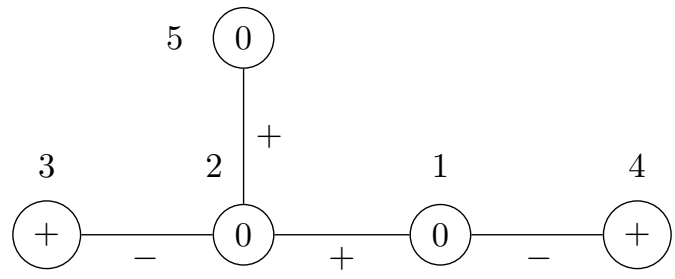

Two signed trees are said to be equivalent if one is obtained from the other after relabeling its vertices. A tree sign pattern matrix is symmetric if each edge of its signed tree is + .

Let $C$ be a square matrix of order $n$. If $\alpha \subseteq\{1,2, \ldots, n\}$, then $C[\alpha]$ is the principal submatrix of $C$ having rows and columns corresponding to the indices in $\alpha$. The inertia of $C$ is the triple $i(C)=$ $\left(i_{+}(C), i_{-}(C), i_{0}(C)\right)$, where $i_{+}(C)$ is the number of eigenvalues of $C$ with positive real part, $i_{-}(C)$ is the number of eigenvalues of $C$ with negative real part and $i_{0}(C)$ is the number of eigenvalues of $C$ with zero real part. A sign pattern matrix $A$ of order $n$ is said to be potentially stable if there exists a $C \in Q(A)$ such that $i(C)=(0, n, 0)$.

For a real number $a$, the function sgn is defined by:

$$
\operatorname{sgn}(a)= \begin{cases}1, & \text { if } a>0 \\ 0, & \text { if } a=0 \\ -1, & \text { if } a<0\end{cases}
$$

We recall from [14] that a sign pattern matrix $A$ of order $n$ allows a properly signed nest if there exists a $C \in Q(A)$ and a permutation matrix $P$ of order $n$ such that

$$
\operatorname{sgn} \operatorname{det}\left(\left(P C P^{T}\right)[\{1,2, \ldots, k\}]\right)=(-1)^{k} \text { for } k=1,2, \ldots, n \text {. }
$$

The following result was given by Johnson et al. [14] in an attempt to obtain a sufficient condition for a tree sign pattern matrix with negative edges to allow a properly signed nest.

[14, Corollary 3.7]. If $A$ is a tree sign pattern matrix in which at least one diagonal entry is negative and every edge is negative (except possibly those with both end vertices negative), and $A$ allows a nonzero determinant, then $A$ allows a properly signed nest.

However, the following example shows that the above result is not true in general. 
EXAMPLE 1.1. Let us consider the tree sign pattern matrix:

$$
A=\left[\begin{array}{lll}
+ & + & 0 \\
- & - & + \\
0 & - & +
\end{array}\right] .
$$

Let $C \in Q(A)$. Then without loss of generality we may assume that

$$
C=\left[\begin{array}{ccc}
a & 1 & 0 \\
-d & -b & 1 \\
0 & -e & c
\end{array}\right],
$$

where $a, b, c, d, e>0$. If $A$ allows a properly signed nest, then we must have

$$
-b<0, \quad e-b c>0 \text { or } d-a b>0 \text { and } a e+c d-a b c<0,
$$

for some $a, b, c, d, e>0$, which is not possible. So $A$ does not allow a properly signed nest.

Remark 1.2. By [15, Fig. 2], $A$ in the above example is not potentially stable.

In Section 2, we give some sufficient conditions for tree sign pattern matrices with all edges negative to allow a properly signed nest.

Johnson et al. [14] proved that if a sign pattern matrix allows a properly signed nest, then it is potentially stable. They also mention that the converse is not true, even for tree sign pattern matrices. In Section 3, we discuss the cases for which the converse is also true and characterize potentially stable star and path sign pattern matrices with all edges negative.

Drew et al. [6] introduced the concept of spectrally arbitrary patterns. A sign pattern matrix $A$ of order $n$ is said to be a spectrally arbitrary pattern if, for any given real monic polynomial $r(x)$ of degree $n$, there is a matrix in $Q(A)$ with characteristic polynomial $r(x)$. In Section 4, we describe all 5-by-5 spectrally arbitrary tree sign pattern matrices with all edges negative.

2. Tree sign pattern matrices with all edges negative that allow properly signed nests. Olesky et al. [17] characterized sign pattern matrices allowing a properly signed nest in terms of another allow problem. Cavalcanti [4] characterized sign pattern matrices allowing a properly signed nest through a relation between two subsequent leading principal minors (after permutation, if required). Using matchings of a graph, we give some sufficient conditions for a sign pattern matrix with all edges negative to allow a properly signed nest. Let us recall the following definitions from [10].

If $G$ is a simple undirected graph with vertex set $V(G)$ and edge set $E(G)$, then the subgraph of $G$ induced by $S \subseteq V(G)$ is the graph with vertex set $S$ and edge set $\{[i, j] \in E(G): i, j \in S\}$. We denote this induced subgraph by $G(S)$. If $S \subseteq V(G)$, then $G-S$ is the subgraph of $G$ obtained from $G$ by deleting the vertices in $S$ and the edges incident to them. A path between two vertices $u, v$ of $G$ is a sequence $\left\{v_{0}, v_{1}, v_{2}, \ldots, v_{k-1}, v_{k}\right\}$ of distinct vertices such that $v_{0}=u, v_{k}=v$, and $\left[v_{i-1}, v_{i}\right] \in E(G)$ for all $i \in\{1,2, \ldots, k\}$. A set of edges in an undirected graph $G$ is called a matching if no two edges in that set have a common end vertex. A perfect matching of a graph $G$ is a matching such that each vertex of $G$ is an end vertex of an edge in that matching. The degree of a vertex $u$, denoted by $\operatorname{deg}(u)$, is the number of distinct vertices of $G$ adjacent to $u$. If $\operatorname{deg}(u)=1$, then $u$ is called pendant. Throughout this paper, $\langle n\rangle$ denotes the set $\{1,2, \ldots, n\}$. 
565 Potentially stable and 5-by-5 spectrally arbitrary tree sign pattern matrices with all edges negative

LEMma 2.1. Let $T$ be a tree with a vertex $v$ such that $T-\{v\}$ has a perfect matching. Then for each vertex $u$ adjacent to $v$, there is a path $\left\{v_{i_{1}}, v_{i_{2}}, \ldots, v_{i_{k-1}}, v_{i_{k}}\right\}$ such that $\operatorname{deg}\left(v_{i_{k}}\right)=1$ and $\operatorname{deg}\left(v_{i_{k-1}}\right)=2$, where $v_{i_{1}}=v$ and $v_{i_{2}}=u$.

Proof. Let $u$ be a vertex adjacent to $v$. Since $T-\{v\}$ has a perfect matching, $u$ is not pendant. We consider a longest path $\left\{v_{i_{1}}, v_{i_{2}}, \ldots, v_{i_{k-1}}, v_{i_{k}}\right\}$ in $T$, where $v_{i_{1}}=v$ and $v_{i_{2}}=u$. Then, $v_{i_{k}}$ must be a pendant vertex. If $\operatorname{deg}\left(v_{i_{k-1}}\right)>2$, then $v_{i_{k-1}}$ must be adjacent to another pendant vertex, say $w$. So we have two pendant vertices $w, v_{i_{k}}$ distinct from $v$ such that $v_{i_{k-1}}$ is adjacent to both $w, v_{i_{k}}$. Then, $T-\{v\}$ cannot have a perfect matching, a contradiction. So $\operatorname{deg}\left(v_{i_{k-1}}\right)=2$.

LEMma 2.2. Let $T$ be a tree with $2 n+1$ vertices and let $v$ be a vertex of $T$. If $T-\{v\}$ has a perfect matching, then we can label the vertices with the numbers from $\langle 2 n+1\rangle$ such that the vertex $v$ is labeled as 1 and for each $s \in\langle 2 n+1\rangle, G(\langle s\rangle)$ has a perfect matching when $s$ is even, and $G(\langle s\rangle)-\{1\}$ has a perfect matching when $s$ is odd.

Proof. We prove this by induction on $n$. For $n=1, T$ is a tree with three vertices. Since $T-\{v\}$ has a perfect matching, $v$ must be a pendant vertex. If we label $v$ as 1 , the vertex adjacent to $v$ as 2 , and the other vertex as 3 , we get the desired result.

Suppose the required result is true for any tree with $2 n-1$ vertices. Let $T$ be a tree with $2 n+1$ vertices, and let $T$ has a vertex $v$ such that $T-\{v\}$ has a perfect matching. Then by Lemma 2.1, there is a path $\left\{v_{i_{1}}, v_{i_{2}}, \ldots, v_{i_{k-1}}, v_{i_{k}}\right\}$ such that $\operatorname{deg}\left(v_{i_{k}}\right)=1$ and $\operatorname{deg}\left(v_{i_{k-1}}\right)=2$, where $v_{i_{1}}=v$ and $v_{i_{k-1}}, v_{i_{k}} \neq v$. So $\tilde{T}=T-\left\{v_{i_{k-1}}, v_{i_{k}}\right\}$ is a tree such that $\tilde{T}-\{v\}$ has a perfect matching. Further, $\tilde{T}$ is a tree with $2 n-1$ vertices. So by induction hypothesis, we can label $\tilde{T}$ such that the vertex $v$ is labeled as 1 ; and for each $s \in\langle 2 n-1\rangle, G(\langle s\rangle)$ has a perfect matching when $s$ is even, and $G(\langle s\rangle)-\{1\}$ has a perfect matching when $s$ is odd. If $v_{i_{k-2}}$ is labeled as $m$ in the above labeling, then label $v_{i_{k-1}}$ as $m+1, v_{i_{k}}$ as $m+2$, and relabel all vertices $j(>m)$ of $\tilde{T}$ as $j+2$.

If $m$ is even, then by induction hypothesis, both $G(\langle m\rangle)$ and $G(\langle m-1\rangle)-\{1\}$ have perfect matchings in $\tilde{T}$. So $G(\langle m+1\rangle)-\{1\}$ has a perfect matching, namely the perfect matching for $G(\langle m-1\rangle)-\{1\}$ in $\tilde{T}$ together with the edge $[m, m+1]$; and $G(\langle m+2\rangle)$ has a perfect matching, namely the perfect matching for $G(\langle m\rangle)$ in $\tilde{T}$ together with the edge $[m+1, m+2]$.

If $m$ is odd, then by induction hypothesis, both $G(\langle m\rangle)-\{1\}$ and $G(\langle m-1\rangle)$ have perfect matchings in $\tilde{T}$. So $G(\langle m+1\rangle)$ has a perfect matching, namely the perfect matching for $G(\langle m-1\rangle)$ in $\tilde{T}$ together with the edge $[m, m+1]$; and $G(\langle m+2\rangle)-\{1\}$ has a perfect matching, namely the perfect matching for $G(\langle m\rangle)-\{1\}$ in $\tilde{T}$ together with the edge $[m+1, m+2]$.

For each odd $s>m+2, G(\langle s\rangle)-\{1\}$ has a perfect matching, namely the perfect matching for $G(\langle s-$ $2\rangle)-\{1\}$ in $\tilde{T}$ together with the edge $[m+1, m+2]$. For each even $s>m+2, G(\langle s\rangle)$ has a perfect matching, namely the perfect matching for $G(\langle s-2\rangle)$ in $\tilde{T}$ together with the edge $[m+1, m+2]$.

For each odd $s \leq m, G(\langle s\rangle)-\{1\}$ has a perfect matching, namely the perfect matching for $G(\langle s\rangle)-\{1\}$ in $\tilde{T}$. For each even $s \leq m, G(\langle s\rangle)$ has a perfect matching, namely the perfect matching for $G(\langle s\rangle)$ in $\tilde{T}$.

Therefore for each $s \in\langle 2 n+1\rangle, G(\langle s\rangle)$ has a perfect matching when $s$ is even, and $G(\langle s\rangle)-\{1\}$ has a perfect matching when $s$ is odd.

Corollary 3.8 of [14] can be rephrased as follows. 
Lemma 2.3 ([14]). If a sign pattern matrix A allows a properly signed nest, then every super-pattern of $A$ also allows a properly signed nest.

The following theorem gives a sufficient condition for a tree sign pattern matrix of odd order with negative edges to allow a properly signed nest.

TheOREM 2.4. Let $A$ be a tree sign pattern matrix of odd order $n$ with all edges negative. If $G(A)$ contains a vertex $v$ such that $G(A)-\{v\}$ has a perfect matching and $a_{v v}=-$, then $A$ allows a properly signed nest.

Proof. Let $B$ be a subpattern of $A$ such that $B$ is a tree sign pattern matrix, $b_{v v}=-$ and $b_{u u}=0$ for all $u \neq v$. By Lemma 2.2, we can label $G(B)$ such that $v$ is labeled as 1 so that for each $s \in\langle n\rangle$, the induced subgraph $G(\langle s\rangle)$ has a perfect matching when $s$ is even, and $G(\langle s\rangle)-\{1\}$ has a perfect matching when $s$ is odd. Let $C \in Q(B)$ be such that $c_{i j}=1,0,-1$ accordingly as $b_{i j}=+, 0,-$. So there exists a permutation matrix $P$ such that $\operatorname{det}\left(\left(P C P^{T}\right)[\langle s\rangle]\right)=(-1)^{s}$ for all $s \in\langle n\rangle$. Thus, $B$ allows a properly signed nest, and therefore by Lemma 2.3, $A$ allows a properly signed nest.

The converse of the above result is not true.

EXAMPLE 2.5. Let us consider a tree sign pattern matrix $A$ with its signed tree as follows:

$$
\begin{gathered}
A=\left[\begin{array}{ccccc}
- & + & 0 & + & 0 \\
- & 0 & + & 0 & + \\
0 & - & + & 0 & 0 \\
- & 0 & 0 & + & 0 \\
0 & - & 0 & 0 & 0
\end{array}\right] \\
C=\left[\begin{array}{ccccc}
-3 & 1 & 0 & 1 & 0 \\
-2 & 0 & 1 & 0 & 1 \\
0 & -1 & 1 & 0 & 0 \\
-2 & 0 & 0 & 1 & 0 \\
0 & -1 & 0 & 0 & 0
\end{array}\right] \in Q(A) .
\end{gathered}
$$

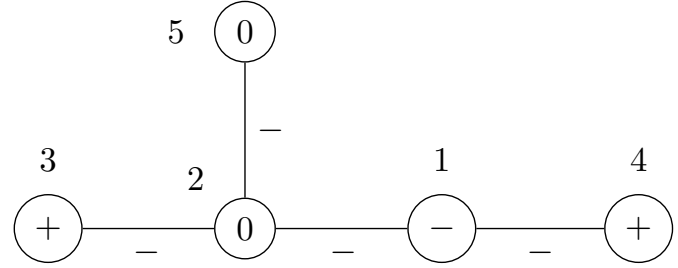

The leading principal minors of $C$ are $-3,2,-1,1$, and -1 . So $A$ allows a properly signed nest. However, $G(A)-\{1\}$ does not have a perfect matching.

Lemma 2.6. If two sign pattern matrices $A_{1}$ and $A_{2}$ allow properly signed nests, then $A_{1} \oplus A_{2}$ allows a properly signed nest.

Proof. Let the orders of $A_{1} A_{2}$ be $r$ and $s$ respectively. Since both $A_{1}$ and $A_{2}$ allow properly signed nests, there exist permutation matrices $P_{1}$ and $P_{2}$ and $C_{1} \in Q\left(A_{1}\right)$ and $C_{2} \in Q\left(A_{2}\right)$ such that

$$
\operatorname{sgn} \operatorname{det}\left(P_{1} C_{1} P_{1}^{T}\right)[\langle t\rangle]=(-1)^{t} \text { for } t=1,2, \ldots, r,
$$

and

$$
\operatorname{sgn} \operatorname{det}\left(P_{2} C_{2} P_{2}^{T}\right)[\langle t\rangle]=(-1)^{t} \text { for } t=1,2, \ldots, s .
$$

Then, sgn $\operatorname{det}\left[\left(P_{1} \oplus P_{2}\right)\left(C_{1} \oplus C_{2}\right)\left(P_{1} \oplus P_{2}\right)^{T}\right][\langle t\rangle]=(-1)^{t}$ for $t=1,2, \ldots, r+s$. Since $C_{1} \oplus C_{2} \in Q\left(A_{1} \oplus A_{2}\right)$, $A_{1} \oplus A_{2}$ allows a properly signed nest. 
567 Potentially stable and 5-by-5 spectrally arbitrary tree sign pattern matrices with all edges negative

The following corollary is a generalization of Theorem 2.4.

COROllary 2.7. Let $A$ be a tree sign pattern matrix with all edges negative. If there exist $k$ vertices $v_{1}, \ldots, v_{k}$ such that $G(A)-\left\{v_{1}, \ldots, v_{k}\right\}$ has a perfect matching and $a_{v_{i} v_{i}}=-$ for $i=1,2, \ldots, k$, then $A$ allows a properly signed nest.

Proof. Suppose there exist vertices $v_{1}, \ldots, v_{k}$ such that $G(A)-\left\{v_{1}, \ldots, v_{k}\right\}$ has a perfect matching. Let $M$ be such a perfect matching. If two vertices, say $v_{k-1}, v_{k}$ are adjacent, then $G(A)-\left\{v_{1}, \ldots, v_{k-2}\right\}$ has a perfect matching $M \cup\left\{\left[v_{k-1}, v_{k}\right]\right\}$. Without loss of generality, we may assume that no two vertices from $\left\{v_{1}, \ldots, v_{k}\right\}$ are adjacent.

We prove this by induction on $k$. For $k=1$, the result is true by Theorem 2.4. Suppose the result is true for $k=m$ and we consider a tree sign pattern matrix $A$ with $k=m+1$.

Let $\mathcal{P}$ be the path in $G(A)$ from $v_{1}$ to $v_{2}$. Then the edge $e$ in $\mathcal{P}$ with an end vertex $v_{1}$ does not belong to $M$. If we delete the edge $e$, then we will have two tree components. Let $\tilde{A}$ be the sign pattern matrix obtained from $A$ by converting the two entries corresponding to the edge $e$ to zero. So there exists a permutation sign pattern matrix $P$ such that $P \tilde{A} P^{T}=A_{1} \oplus A_{2}$, where both $A_{1}$ and $A_{2}$ allow properly signed nests, by induction hypothesis. Therefore, by Lemma 2.6, $A_{1} \oplus A_{2}$ allows a properly signed nest, and thus by Lemma 2.3, $A$ allows a properly signed nest.

The following theorem gives a sufficient condition for a tree sign pattern matrix of even order with negative edges to allow a properly signed nest.

THEOREM 2.8. Let $A$ be a tree sign pattern matrix of even order with all edges negative. If $A$ has at least one negative diagonal entry and $G(A)$ has a perfect matching, then $A$ allows a properly signed nest.

Proof. Let $v$ be a vertex such that $a_{v v}=-$. If $B$ is a subpattern of $A$ such that $B$ is a tree sign pattern matrix, $b_{v v}=-$ and $b_{u u}=0$ for all $u \neq v$, then $G(B)$ also has a perfect matching. Let $M$ be a perfect matching of $G(B)$. Consider a path $\left\{v_{1}, v_{2}, \ldots, v_{2 k}\right\}$ in $G(B)$ such that $v_{1}=v, \operatorname{deg}\left(v_{2 k}\right)=1$ and

$$
S=\left\{\left[v_{2 i-1}, v_{2 i}\right]: 1 \leq i \leq k\right\} \subseteq M
$$

Let $T=G(B)-\left\{v_{2 k}\right\}$. Then $(M \backslash S) \cup\left\{\left[v_{2 i}, v_{2 i+1}\right]: 1 \leq i \leq k-1\right\}$ is a perfect matching for $T-\{v\}$. Therefore, by Theorem 2.4, $B\left(\left\{v_{2 k}\right\}\right)$ allows a properly signed nest and $\operatorname{sign} \operatorname{det} B\left(\left\{v_{2 k}\right\}\right)=-$. Again, $\operatorname{sign} \operatorname{det}(B)=+$. Therefore, $B$ allows a properly signed nest. Hence, by Lemma 2.3, $A$ allows a properly signed nest.

The converse of the above result is not true.

ExAMPLE 2.9. Let us consider a tree sign pattern matrix $A$ with its signed tree as follows:

$$
A=\left[\begin{array}{cccc}
0 & - & + & + \\
+ & 0 & 0 & 0 \\
- & 0 & - & 0 \\
- & 0 & 0 & -
\end{array}\right]
$$

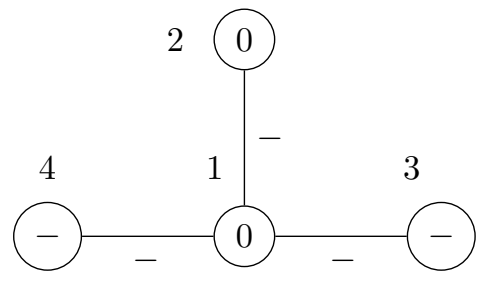

By Corollary 2.7, $A$ allows a properly signed nest. However, $G(A)$ does not have a perfect matching. 
3. Potentially stable tree sign pattern matrices with all edges negative. Characterization of potentially stable sign pattern matrices has been a long-standing open problem. Gao and Li [7] gave a necessary and sufficient condition for potentially stable star sign pattern matrices. Few methods to construct higher-order potentially stable sign pattern matrices from lower-order potentially stable sign pattern matrices are given in $[2,8]$. Cavers [5] gave few methods to identify some sign pattern matrices which are not potentially stable.

For a tree sign pattern matrix $A$, the symmetric factorization of $A$ is $A=\tilde{S} \tilde{A}$, where $\tilde{S}$ is a signature sign pattern matrix with the $(1,1)$ entry + and $\tilde{A}$ is a symmetric tree sign pattern matrix. Let $i_{+}(S)$ denotes the number of + of a signature sign pattern matrix $S$. The following result by Jeffries and Johnson [13] is very useful in identifying some tree sign pattern matrices which are not potentially stable.

Lemma 3.1 ([13]). Let $A=\tilde{S} \tilde{A}$ be the symmetric factorization of an $n \times n$ tree sign pattern matrix $A$. If $A$ is potentially stable, then there is a symmetric matrix $\tilde{C} \in Q(\tilde{A})$ such that $i_{+}(\tilde{C})=n-i_{+}(\tilde{S})$.

The following result is given by Johnson et al. [14].

Lemma 3.2 ([14]). If a sign pattern matrix A allows a properly signed nest, then $A$ is potentially stable.

As we discussed earlier, the converse is not true, even for tree sign pattern matrices. Now we discuss the cases for which the converse is also true. Johnson and Summers [15] proved that a super-pattern of a potentially stable sign pattern matrix is also a potentially stable sign pattern matrix.

Lemma $3.3([15])$. Suppose that $B$ is a potentially stable $n \times n$ sign pattern matrix, and suppose that $B$ is a subpattern of the $n \times n$ sign pattern matrix $A$. Then $A$ is also potentially stable.

The following result characterizes all potentially stable path sign pattern matrices with all edges negative.

THEOREM 3.4. If $A$ is a path sign pattern matrix of order $n$ with all edges negative and $G(A)$ is the graph of $A$ with vertices $1,2, \ldots, n$ such that $i$ is adjacent to $i+1$ for $i=1,2, \ldots, n-1$, then the following statements are equivalent.

1. A is potentially stable.

2. A allows a properly signed nest.

3. Exactly one of the following statements is true.

(a) $n$ is even and $A$ has a negative diagonal entry.

(b) $n$ is odd and the $i$-th diagonal entry of $A$ is negative for some odd $i$.

Proof. Theorems 2.4 and 2.8 imply $3 \Rightarrow 2$. By Lemma $3.2,2 \Rightarrow 1$. Now we show $1 \Rightarrow 3$.

$A$ is potentially stable implies that $A$ has a negative diagonal entry. So $1 \Rightarrow 3$ when $n$ is even. If $n$ is odd, then suppose $a_{i i} \neq-$ for all odd $i$. Consider a path sign pattern matrix $B$ of order $n$ such that $A$ is a subpattern of $B$ and the $(2 i-1)$-th diagonal entry of $B$ is positive for all $i$ with $1 \leq i \leq \frac{n+1}{2}$. Let $B=\tilde{S} \tilde{B}$ be the symmetric factorization of $B$. Since the number of vertices at even distance from the vertex 1 including itself is $\frac{n+1}{2}, i_{+}(\tilde{S})=\frac{n+1}{2}$. If $B$ is potentially stable, then by Lemma 3.1, there exists a symmetric matrix $\tilde{C} \in Q(\tilde{B})$ such that $i_{+}(\tilde{C})=n-i_{+}(\tilde{S})=\frac{n-1}{2}$. But $\tilde{B}$ has a principal submatrix, which is a direct sum of $\frac{n+1}{2}[+]$ s. So by Cauchy's interlacing theorem for real symmetric matrices ([11, Theorem 4.3.17]), $i_{+}(\tilde{X}) \geq \frac{n+1}{2}$ for any symmetric matrix $\tilde{X} \in Q(\tilde{B})$. Thus, $B$ is not potentially stable, and therefore by Lemma 3.3, $A$ is not potentially stable, which is a contradiction. So $A$ is a potentially stable sign pattern matrix of order $n$ implies that the $i$-th diagonal entry of $A$ is negative for some odd $i$. Therefore, $1 \Rightarrow 3$. 
569 Potentially stable and 5-by-5 spectrally arbitrary tree sign pattern matrices with all edges negative

The following result characterizes all potentially stable star sign pattern matrices with all edges negative.

THEOREM 3.5. If $A$ is a star sign pattern matrix of order $n \geq 2$ with all edges negative, then the following statements are equivalent.

1. A is potentially stable.

2. A allows a properly signed nest.

3. A diagonal entry corresponding to at most one pendant vertex of $G(A)$ is nonnegative.

Proof. For $n=2$, the potentially stable star sign pattern matrices (up to equivalence) with all edges negative are one of the following sign pattern matrices:

$$
\left[\begin{array}{cc}
- & + \\
- & 0
\end{array}\right],\left[\begin{array}{cc}
+ & + \\
- & -
\end{array}\right],\left[\begin{array}{ll}
- & + \\
- & -
\end{array}\right]
$$

Each of these sign pattern matrices satisfies all the above conditions. So the result is true for $n=2$.

For $n \geq 3$, Corollary 2.7 implies that $3 \Rightarrow 2$. Again by Lemma $3.2,2 \Rightarrow 1$. Now we show $1 \Rightarrow 3$.

If condition 3 is not true, then at least two diagonal entries corresponding to pendant vertices of $G(A)$ are nonnegative. Let $B$ be a star sign pattern matrix of order $n$ such that $A$ is a subpattern of $B$ and two diagonal entries of $B$ corresponding to pendant vertices of $G(A)$ are + . Without loss of generality, we may assume that one of these pendant vertices is labeled as 1 . Let $B=\tilde{S} \tilde{B}$ be the symmetric factorization of $B$. Since the number of vertices at even distance from the vertex 1 including itself is $n-1, i_{+}(\tilde{S})=n-1$. If $B$ is potentially stable, then by Lemma 3.1, there exists a symmetric matrix $\tilde{C} \in Q(\tilde{B})$ such that $i_{+}(\tilde{C})=n-i_{+}(\tilde{S})=1$. But $\tilde{B}$ has a principal submatrix $[+] \oplus[+]$. So by Cauchy's interlacing theorem for real symmetric matrices, $i_{+}(\tilde{X}) \geq 2$ for any symmetric matrix $\tilde{X} \in Q(\tilde{B})$. Thus, $B$ is not potentially stable. So by Lemma $3.3, A$ is not potentially stable, which is a contradiction. Therefore, $1 \Rightarrow 3$.

We get from Lemma 3.2 that if a sign pattern matrix allows a properly signed nest, then it is potentially stable. Since from Theorems 3.4 and 3.5, we get that for path and star sign pattern matrices with all edges negative, potential stability implies the existence of a properly signed nest, we believe that the same is true for all tree sign pattern matrices with all edges negative.

CONJeCture 3.6. A tree sign pattern matrix A with all edges negative is potentially stable if and only if it allows a properly signed nest.

If $A$ has exactly one nonzero diagonal entry, then the conjecture is true by [14, Theorem 4.2]. We do not have a complete answer to the above conjecture as yet; however, the following theorem shows that the above conjecture is true for tree sign pattern matrices up to order 6 .

TheOREm 3.7. Let $A$ be a tree sign pattern matrix of order at most 6 with all edges negative. Then $A$ is potentially stable if and only if $A$ allows a properly signed nest.

Proof. Let $A$ be a tree sign pattern matrix of order $n$ with all edges negative. By Lemma 3.2, if a sign pattern matrix allows a properly signed nest, then it is potentially stable. So we need to show that if $A$ is potentially stable, then $A$ allows a properly signed nest.

Case I: $n \leq 4$. In this case, $G(A)$ is either a path or a star. So the result is true by Theorems 3.4 and 3.5 , respectively. 
Case II: $n=5$. If $G(A)$ is a path or a star, then the result is true by Theorems 3.4 and 3.5, respectively. Suppose $G(A)$ is neither a star nor a path. Then $A$ with its signed tree is as follows:

$$
A=\left[\begin{array}{ccccc}
a_{1} & + & + & + & 0 \\
- & a_{2} & 0 & 0 & 0 \\
- & 0 & a_{3} & 0 & 0 \\
- & 0 & 0 & a_{4} & + \\
0 & 0 & 0 & - & a_{5}
\end{array}\right]
$$

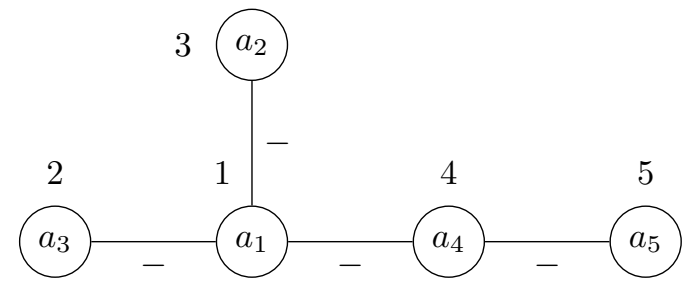

The symmetric factorization of $A$ is

$$
A=\left[\begin{array}{ccccc}
a_{1} & + & + & + & 0 \\
- & a_{2} & 0 & 0 & 0 \\
- & 0 & a_{3} & 0 & 0 \\
- & 0 & 0 & a_{4} & + \\
0 & 0 & 0 & - & a_{5}
\end{array}\right]=\left[\begin{array}{ccccc}
+ & 0 & 0 & 0 & 0 \\
0 & - & 0 & 0 & 0 \\
0 & 0 & - & 0 & 0 \\
0 & 0 & 0 & - & 0 \\
0 & 0 & 0 & 0 & +
\end{array}\right]\left[\begin{array}{ccccc}
a_{1} & + & + & + & 0 \\
+ & -a_{2} & 0 & 0 & 0 \\
+ & 0 & -a_{3} & 0 & 0 \\
+ & 0 & 0 & -a_{4} & - \\
0 & 0 & 0 & - & a_{5}
\end{array}\right]=\tilde{S} \tilde{A} .
$$

If $A$ is potentially stable, then by Lemma 3.1 , there exists a symmetric matrix $\tilde{C} \in Q(\tilde{A})$ such that $i_{+}(\tilde{C})=$ $5-i_{+}(\tilde{S})=3$ and thus $i_{-}(\tilde{C}) \leq 2$.

Case II(1). If $a_{2}, a_{3}, a_{4}=+$, then the eigenvalues of $\tilde{X}[\{2,3,4\}]$ are negative for all $\tilde{X} \in Q(\tilde{A})$. So by Cauchy's interlacing theorem, $i_{-}(\tilde{X}) \geq 3$ for all symmetric matrix $\tilde{X} \in Q(\tilde{A})$, a contradiction. Therefore, $A$ is not potentially stable. Hence, by Lemma $3.3, A$ is not potentially stable if, $a_{2}, a_{3}, a_{4} \in$ $\{0,+\}$.

Case II (2). If $a_{2}, a_{3}=+, a_{5}=-$, then the eigenvalues of $\tilde{X}[\{2,3,5\}]$ are negative for all $\tilde{X} \in Q(\tilde{A})$. So by Cauchy's interlacing theorem, $i_{-}(\tilde{X}) \geq 3$ for all symmetric matrix $\tilde{X} \in Q(\tilde{A})$, a contradiction. Therefore, $A$ is not potentially stable. Hence, by Lemma 3.3, $A$ is not potentially stable if, $a_{2}, a_{3} \in$ $\{0,+\}$ and $a_{5} \in\{0,-\}$.

Now $a_{2}, a_{3}$ can be interchanged by a permutation similarity, and the potential stability of a sign pattern matrix is not affected by permutation similarity. Suppose $A$ is potentially stable. Then by Case II(1), at least one of $a_{2}, a_{3}, a_{4}$ is -. If $a_{2}=-$ or $a_{3}=-$, then $A$ is permutation similar to some super-pattern of

$$
A_{1}=\left[\begin{array}{ccccc}
0 & + & + & + & 0 \\
- & - & 0 & 0 & 0 \\
- & 0 & 0 & 0 & 0 \\
- & 0 & 0 & 0 & + \\
0 & 0 & 0 & - & 0
\end{array}\right]
$$

If $a_{4}=-$ and $A$ is not a super-pattern of $A_{1}$, then $a_{2}, a_{3} \in\{0,+\}$ and thus by Case II (2), $a_{5}=+$. Further, $A$ is potentially stable implies $A$ allows nonsingularity and thus at least one of $a_{2}$ and $a_{3}$ is + . Therefore, $A$ is permutation similar to some super-pattern of

$$
A_{2}=\left[\begin{array}{ccccc}
0 & + & + & + & 0 \\
- & + & 0 & 0 & 0 \\
- & 0 & 0 & 0 & 0 \\
- & 0 & 0 & - & + \\
0 & 0 & 0 & - & +
\end{array}\right]
$$


571 Potentially stable and 5-by-5 spectrally arbitrary tree sign pattern matrices with all edges negative

Now $A_{1}$ allows a properly signed nest by Theorem 2.4. Again $A_{2}$ is permutation similar to the sign pattern matrix in Example 2.5, and thus $A_{2}$ allows a properly signed nest. Therefore, by Lemma $2.3, A$ is potentially stable implies $A$ allows a properly signed nest.

Case III: $n=6$. If $G(A)$ is a path or a star, then the result is true by Theorems 3.4 and 3.5, respectively. Suppose $G(A)$ is neither a star nor a path. There are four nonequivalent such signed trees, which are listed below:
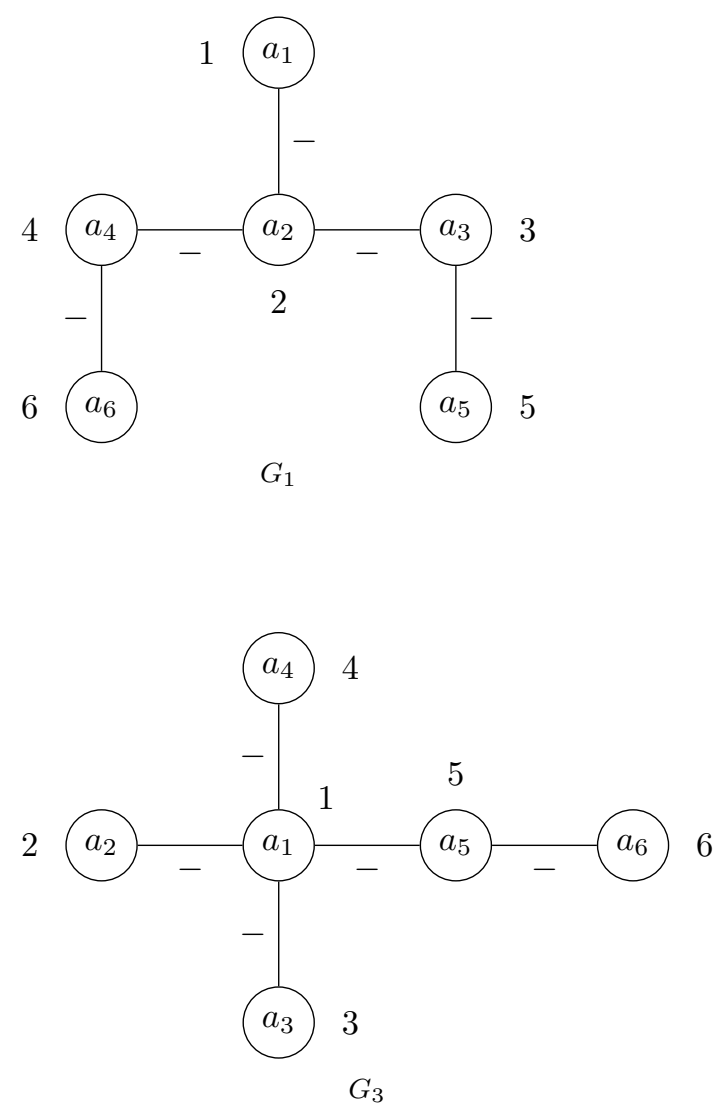
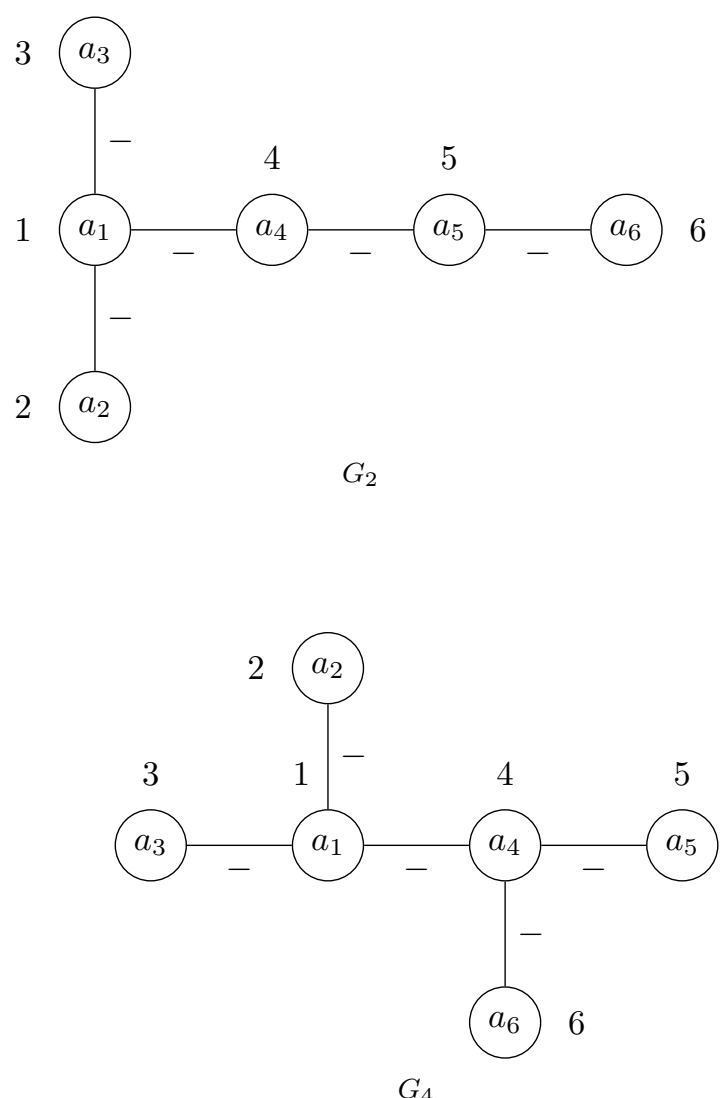

1. Let $G(A)$ be equivalent to $G_{1}$. If $A$ is potentially stable, then $A$ has a negative diagonal entry. So by Theorem 2.8, $A$ is potentially stable implies $A$ allows a properly signed nest.

2. If $G(A)$ is equivalent to $G_{2}$, then we can consider

$$
A=\left[\begin{array}{cccccc}
a_{1} & + & + & + & 0 & 0 \\
- & a_{2} & 0 & 0 & 0 & 0 \\
- & 0 & a_{3} & 0 & 0 & 0 \\
- & 0 & 0 & a_{4} & + & 0 \\
0 & 0 & 0 & - & a_{5} & + \\
0 & 0 & 0 & 0 & - & a_{6}
\end{array}\right]
$$


The symmetric factorization of $A$ is

$$
A=\left[\begin{array}{cccccc}
+ & 0 & 0 & 0 & 0 & 0 \\
0 & - & 0 & 0 & 0 & 0 \\
0 & 0 & - & 0 & 0 & 0 \\
0 & 0 & 0 & - & 0 & 0 \\
0 & 0 & 0 & 0 & + & 0 \\
0 & 0 & 0 & 0 & 0 & -
\end{array}\right]\left[\begin{array}{cccccc}
a_{1} & + & + & + & 0 & 0 \\
+ & -a_{2} & 0 & 0 & 0 & 0 \\
+ & 0 & -a_{3} & 0 & 0 & 0 \\
+ & 0 & 0 & -a_{4} & - & 0 \\
0 & 0 & 0 & - & a_{5} & + \\
0 & 0 & 0 & 0 & + & -a_{6}
\end{array}\right]=\tilde{S} \tilde{A}
$$

If $A$ is potentially stable, then by Lemma 3.1 , there exists a symmetric matrix $\tilde{C} \in Q(\tilde{A})$ such that $i_{+}(\tilde{C})=6-i_{+}(\tilde{S})=4$ and thus $i_{-}(\tilde{C}) \leq 2$.

Case III(2.1) If any three of $a_{2}, a_{3}, a_{4}$, and $a_{6}$ are + , then by Cauchy's interlacing theorem, $i_{-}(\tilde{X}) \geq 3$ for all symmetric matrix $\tilde{X} \in Q(\tilde{A})$, a contradiction. Therefore, $A$ is not potentially stable. Hence, by Lemma $3.3, A$ is not potentially stable if, any three of $a_{2}, a_{3}, a_{4}$, and $a_{6}$ are in $\{0,+\}$.

Case III(2.2) If $a_{2}, a_{3}=+, a_{5}=-$, then by Cauchy's interlacing theorem, $i_{-}(\tilde{X}) \geq 3$ for all symmetric matrix $\tilde{X} \in Q(\tilde{A})$, a contradiction. Therefore, $A$ is not potentially stable. Hence, by Lemma 3.3, $A$ is not potentially stable if, $a_{2}, a_{3} \in\{0,+\}$ and $a_{5} \in\{0,-\}$.

Now $a_{2}$ and $a_{3}$ can be interchanged by a permutation similarity, and the potential stability of a sign pattern matrix is not affected by permutation similarity. Suppose $A$ is potentially stable. Then from Case III(2.1), at least one of $\left(a_{2}, a_{4}\right),\left(a_{2}, a_{6}\right),\left(a_{3}, a_{4}\right),\left(a_{3}, a_{6}\right),\left(a_{2}, a_{3}\right),\left(a_{4}, a_{6}\right)$ is $(-,-)$. If at least one of $\left(a_{2}, a_{4}\right),\left(a_{2}, a_{6}\right),\left(a_{3}, a_{4}\right),\left(a_{3}, a_{6}\right),\left(a_{2}, a_{3}\right)$ is $(-,-)$, then $A$ is permutation similar to some super-pattern of one among

$$
\begin{gathered}
A_{21}=\left[\begin{array}{cccccc}
0 & + & + & + & 0 & 0 \\
- & - & 0 & 0 & 0 & 0 \\
- & 0 & 0 & 0 & 0 & 0 \\
- & 0 & 0 & - & + & 0 \\
0 & 0 & 0 & - & 0 & + \\
0 & 0 & 0 & 0 & - & 0
\end{array}\right], A_{22}=\left[\begin{array}{cccccc}
0 & + & + & + & 0 & 0 \\
- & - & 0 & 0 & 0 & 0 \\
- & 0 & 0 & 0 & 0 & 0 \\
- & 0 & 0 & 0 & + & 0 \\
0 & 0 & 0 & - & 0 & + \\
0 & 0 & 0 & 0 & - & -
\end{array}\right], \\
A_{23}=\left[\begin{array}{cccccc}
0 & + & + & + & 0 & 0 \\
- & - & 0 & 0 & 0 & 0 \\
- & 0 & - & 0 & 0 & 0 \\
- & 0 & 0 & 0 & + & 0 \\
0 & 0 & 0 & - & 0 & + \\
0 & 0 & 0 & 0 & - & 0
\end{array}\right] .
\end{gathered}
$$

If $\left(a_{4}, a_{6}\right)=(-,-)$ and $A$ is not a super-pattern of one among $A_{21}, A_{22}, A_{23}$, then $a_{2}, a_{3} \in\{0,+\}$ and thus by Case III (2.2), $a_{5}=+$. Further, $A$ is potentially stable implies $A$ allows nonsingularity and thus at least one of $a_{2}, a_{3}$ is + . Therefore $A$ is permutation similar to some super-pattern of

$$
A_{24}=\left[\begin{array}{cccccc}
0 & + & + & + & 0 & 0 \\
- & + & 0 & 0 & 0 & 0 \\
- & 0 & 0 & 0 & 0 & 0 \\
- & 0 & 0 & - & + & 0 \\
0 & 0 & 0 & - & + & + \\
0 & 0 & 0 & 0 & - & -
\end{array}\right]
$$


573 Potentially stable and 5-by-5 spectrally arbitrary tree sign pattern matrices with all edges negative

Now by Corollary 2.7, $A_{21}, A_{22}, A_{23}$ allow properly signed nests. Further, $A_{24}(\{6\})$ allows a properly signed nest, since it is permutation similar to the sign pattern matrix in Example 2.5. Thus by Lemmas 2.3 and 2.6, $A_{24}$ allows a properly signed nest. Therefore by Lemma 2.3, $A$ is potentially stable implies $A$ allows a properly signed nest.

3. If $G(A)$ is equivalent to $G_{3}$, then we can consider

$$
A=\left[\begin{array}{cccccc}
a_{1} & + & + & + & + & 0 \\
- & a_{2} & 0 & 0 & 0 & 0 \\
- & 0 & a_{3} & 0 & 0 & 0 \\
- & 0 & 0 & a_{4} & 0 & 0 \\
- & 0 & 0 & 0 & a_{5} & + \\
0 & 0 & 0 & 0 & - & a_{6}
\end{array}\right]
$$

The symmetric factorization of $A$ is

$$
A=\left[\begin{array}{cccccc}
+ & 0 & 0 & 0 & 0 & 0 \\
0 & - & 0 & 0 & 0 & 0 \\
0 & 0 & - & 0 & 0 & 0 \\
0 & 0 & 0 & - & 0 & 0 \\
0 & 0 & 0 & 0 & - & 0 \\
0 & 0 & 0 & 0 & 0 & +
\end{array}\right]\left[\begin{array}{cccccc}
a_{1} & + & + & + & + & 0 \\
+ & -a_{2} & 0 & 0 & 0 & 0 \\
+ & 0 & -a_{3} & 0 & 0 & 0 \\
+ & 0 & 0 & -a_{4} & 0 & 0 \\
+ & 0 & 0 & 0 & -a_{5} & - \\
0 & 0 & 0 & 0 & - & a_{6}
\end{array}\right]=\tilde{S} \tilde{A}
$$

If $A$ is potentially stable, then by Lemma 3.1, there exists a symmetric matrix $\tilde{C} \in Q(\tilde{A})$ such that $i_{+}(\tilde{C})=6-i_{+}(\tilde{S})=4$ and thus $i_{-}(\tilde{C}) \leq 2$.

Case III (3.1) If any three of $a_{2}, a_{3}, a_{4}, a_{5}$ are + , then by Cauchy's interlacing theorem, $i_{-}(\tilde{X}) \geq 3$ for all symmetric matrix $\tilde{X} \in Q(\tilde{A})$, a contradiction. Therefore $A$ is not potentially stable. Hence by Lemma 3.3, $A$ is not potentially stable if, any three of $a_{2}, a_{3}, a_{4}, a_{5}$ are in $\{0,+\}$.

Case III(3.2) If any two of $a_{2}, a_{3}, a_{4}$ are + and $a_{6}=-$, then by Cauchy's interlacing theorem, $i_{-}(\tilde{X}) \geq 3$ for all symmetric matrix $\tilde{X} \in Q(\tilde{A})$, a contradiction. Therefore $A$ is not potentially stable. Hence by Lemma $3.3, A$ is not potentially stable if, any two of $a_{2}, a_{3}, a_{4}$ are in $\{0,+\}$ and $a_{6} \in\{0,-\}$.

Now $a_{2}, a_{3}, a_{4}$ can be permuted by a permutation similarity, and the potential stability of a sign pattern matrix is not affected by permutation similarity. Suppose $A$ is potentially stable. Then by Case III (3.1), at least one of $\left(a_{2}, a_{3}\right),\left(a_{2}, a_{4}\right),\left(a_{3}, a_{4}\right),\left(a_{2}, a_{5}\right),\left(a_{3}, a_{5}\right),\left(a_{4}, a_{5}\right)$ is $(-,-)$. If at least one of $\left(a_{2}, a_{3}\right),\left(a_{2}, a_{4}\right),\left(a_{3}, a_{4}\right)$ is $(-,-)$, then $A$ is permutation similar to some super-pattern of

$$
A_{31}=\left[\begin{array}{cccccc}
0 & + & + & + & + & 0 \\
- & - & 0 & 0 & 0 & 0 \\
- & 0 & - & 0 & 0 & 0 \\
- & 0 & 0 & 0 & 0 & 0 \\
- & 0 & 0 & 0 & 0 & + \\
0 & 0 & 0 & 0 & - & 0
\end{array}\right]
$$

If at least one of $\left(a_{2}, a_{5}\right),\left(a_{3}, a_{5}\right),\left(a_{4}, a_{5}\right)$ is $(-,-)$ and $A$ is not a super-pattern of $A_{31}$, then exactly one of $a_{2}, a_{3}, a_{4}$ is - and thus by Case $\operatorname{III}(3.2), a_{6}=+$. Further, $A$ is potentially stable implies 
$A$ allows nonsingularity and thus at least one of $a_{2}, a_{3}, a_{4}$ is + . Therefore $A$ is permutation similar to some super-pattern of

$$
A_{32}=\left[\begin{array}{cccccc}
0 & + & + & + & + & 0 \\
- & + & 0 & 0 & 0 & 0 \\
- & 0 & - & 0 & 0 & 0 \\
- & 0 & 0 & 0 & 0 & 0 \\
- & 0 & 0 & 0 & - & + \\
0 & 0 & 0 & 0 & - & +
\end{array}\right]
$$

Now by Corollary 2.7, $A_{31}$ allows a properly signed nest. Further, $A_{32}(\{3\})$ allows a properly signed nest, since it is permutation similar to the sign pattern matrix in Example 2.5. Thus by Lemmas 2.3 and 2.6, $A_{32}$ allows a properly signed nest. Therefore by Lemma 2.3, $A$ is potentially stable implies $A$ allows a properly signed nest.

4. If $G(A)$ is equivalent to $G_{4}$, then we can consider

$$
A=\left[\begin{array}{cccccc}
a_{1} & + & + & + & 0 & 0 \\
- & a_{2} & 0 & 0 & 0 & 0 \\
- & 0 & a_{3} & 0 & 0 & 0 \\
- & 0 & 0 & a_{4} & + & + \\
0 & 0 & 0 & - & a_{5} & 0 \\
0 & 0 & 0 & - & 0 & a_{6}
\end{array}\right]
$$

The symmetric factorization of $A$ is

$$
A=\left[\begin{array}{cccccc}
+ & 0 & 0 & 0 & 0 & 0 \\
0 & - & 0 & 0 & 0 & 0 \\
0 & 0 & - & 0 & 0 & 0 \\
0 & 0 & 0 & - & 0 & 0 \\
0 & 0 & 0 & 0 & + & 0 \\
0 & 0 & 0 & 0 & 0 & +
\end{array}\right]\left[\begin{array}{cccccc}
a_{1} & + & + & + & 0 & 0 \\
+ & -a_{2} & 0 & 0 & 0 & 0 \\
+ & 0 & -a_{3} & 0 & 0 & 0 \\
+ & 0 & 0 & -a_{4} & - & - \\
0 & 0 & 0 & - & a_{5} & 0 \\
0 & 0 & 0 & - & 0 & a_{6}
\end{array}\right]=\tilde{S} \tilde{A} .
$$

If $A$ is potentially stable, then by Lemma 3.1, there exists a symmetric matrix $\tilde{C} \in Q(\tilde{A})$ such that $i_{+}(\tilde{C})=6-i_{+}(\tilde{S})=3$ and thus $i_{-}(\tilde{C}) \leq 3$.

Case III(4.1) If $a_{2}, a_{3}=+, a_{5}, a_{6}=-$, then by Cauchy's interlacing theorem, $i_{-}(\tilde{X}) \geq 4$ for all symmetric matrix $\tilde{X} \in Q(\tilde{A})$, a contradiction. Therefore $A$ is not potentially stable. Hence by Lemma 3.3, $A$ is not potentially stable if, $a_{2}, a_{3} \in\{0,+\}$ and $a_{5}, a_{6} \in\{0,-\}$.

Case III(4.2) If $a_{2}, a_{3}=-, a_{5}, a_{6}=+$, then by Cauchy's interlacing theorem, $i_{+}(\tilde{X}) \geq 4$ for all symmetric matrix $\tilde{X} \in Q(\tilde{A})$, a contradiction. Therefore $A$ is not potentially stable. Hence by Lemma $3.3, A$ is not potentially stable if, $a_{2}, a_{3} \in\{0,-\}$ and $a_{5}, a_{6} \in\{0,+\}$.

Now $a_{2}, a_{3}$ can be interchanged, $a_{5}, a_{6}$ can be interchanged, and $a_{1}, a_{2}, a_{3}$ can be interchanged with $a_{4}, a_{5}, a_{6}$ respectively by a permutation similarity, and the potential stability of a sign pattern matrix is not affected by permutation similarity. Suppose $A$ is potentially stable. Then by Case III(4.1) and Case III (4.2), at least one of $\left(a_{2}, a_{5}\right),\left(a_{2}, a_{6}\right),\left(a_{3}, a_{5}\right)$, and $\left(a_{3}, a_{6}\right)$ is either $(-,-)$ or $(+,+)$. 
Electronic Journal of Linear Algebra, ISSN 1081-3810

575 Potentially stable and 5-by-5 spectrally arbitrary tree sign pattern matrices with all edges negative

If at least one of $\left(a_{2}, a_{5}\right),\left(a_{2}, a_{6}\right),\left(a_{3}, a_{5}\right)$, and $\left(a_{3}, a_{6}\right)$ is $(-,-)$, then $A$ is permutation similar to some super-pattern of

$$
A_{41}=\left[\begin{array}{cccccc}
0 & + & + & + & 0 & 0 \\
- & - & 0 & 0 & 0 & 0 \\
- & 0 & 0 & 0 & 0 & 0 \\
- & 0 & 0 & 0 & + & + \\
0 & 0 & 0 & - & - & 0 \\
0 & 0 & 0 & - & 0 & 0
\end{array}\right]
$$

If at least one of $\left(a_{2}, a_{5}\right),\left(a_{2}, a_{6}\right),\left(a_{3}, a_{5}\right)$, and $\left(a_{3}, a_{6}\right)$ is $(+,+)$ and $A$ is not a super-pattern of $A_{41}$, then $A$ is permutation similar to some super-pattern of one among

$$
A_{42}=\left[\begin{array}{cccccc}
0 & + & + & + & 0 & 0 \\
- & + & 0 & 0 & 0 & 0 \\
- & 0 & - & 0 & 0 & 0 \\
- & 0 & 0 & 0 & + & + \\
0 & 0 & 0 & - & + & 0 \\
0 & 0 & 0 & - & 0 & 0
\end{array}\right], \quad A_{43}=\left[\begin{array}{cccccc}
- & + & + & + & 0 & 0 \\
- & + & 0 & 0 & 0 & 0 \\
- & 0 & 0 & 0 & 0 & 0 \\
- & 0 & 0 & 0 & + & + \\
0 & 0 & 0 & - & + & 0 \\
0 & 0 & 0 & - & 0 & 0
\end{array}\right],
$$

since $A$ is potentially stable implies $A$ has a - on the diagonal.

Now by Corollary 2.7, $A_{41}$ allows a properly signed nest. Since the leading principal minors of $P C P^{T}$ are $-1,2,-1,1,-1$, and 1 , where

$$
C=\left[\begin{array}{cccccc}
0 & 1 & 1 & 1 & 0 & 0 \\
-3 & 2 & 0 & 0 & 0 & 0 \\
-2 & 0 & -1 & 0 & 0 & 0 \\
-1 & 0 & 0 & 0 & 1 & 1 \\
0 & 0 & 0 & -1 & 1 & 0 \\
0 & 0 & 0 & -1 & 0 & 0
\end{array}\right] \in Q\left(A_{42}\right) \quad \text { and } P=\left[\begin{array}{cccccc}
0 & 0 & 1 & 0 & 0 & 0 \\
1 & 0 & 0 & 0 & 0 & 0 \\
0 & 0 & 0 & 1 & 0 & 0 \\
0 & 0 & 0 & 0 & 1 & 0 \\
0 & 1 & 0 & 0 & 0 & 0 \\
0 & 0 & 0 & 0 & 0 & 1
\end{array}\right]
$$

$A_{42}$ allows a properly signed nest. Further, $A_{43}(\{3\})$ allows a properly signed nest, since it is permutation similar to the sign pattern matrix in Example 2.5. Since $\operatorname{det} A_{43}=+, A_{43}$ allows a properly signed nest. Therefore, by Lemma $2.3, A$ is potentially stable implies $A$ allows a properly signed nest.

4. 5-by-5 spectrally arbitrary tree sign pattern matrices with all edges negative. A sign pattern matrix $A$ of order $n$ is potentially nilpotent if there exists a $C \in Q(A) \operatorname{such} \operatorname{that} \operatorname{det}(z I-C)=z^{n}$, where $I$ is an identity matrix. In this case, $C$ is a nilpotent realization of $A$. Drew et al. [6] had introduced the following method to find spectrally arbitrary sign pattern matrices.

Nilpotent-Jacobian method. To show that an $n$-by- $n$ irreducible pattern $Y$ is a spectrally arbitrary pattern, consider $X \in Q(Y)$ with its nonzero entries specified by positive parameters $x_{0}, x_{1}, \ldots, x_{k}$. If $X$ has a nilpotent realization $\hat{X}$ with $\left(x_{0}, x_{1}, \ldots, x_{k}\right)=\left(\hat{x}_{0}, \hat{x}_{1}, \ldots, \hat{x}_{k}\right)$, and there are $n x_{i}$ s, say $x_{0}, x_{1}, \ldots, x_{n-1}$, such that the Jacobian of the coefficients of $z^{n-1}, z^{n-2}, \ldots, z^{1}, z^{0}$ in $\operatorname{det}(z I-X)$ with respect to $x_{0}, x_{1}, \ldots, x_{n-1}$ is nonzero when evaluated at $\left(\hat{x}_{0}, \hat{x}_{1}, \ldots, \hat{x}_{k}\right)$, then any super-pattern of $Y$ is a spectrally arbitrary pattern.

MacGillivray et al. [16] identified all spectrally arbitrary star sign pattern matrices. All spectrally arbitrary sign pattern matrices up to order 3 were determined by Britz et al. [3]. Arav et al. [1] characterized all 4-by-4 spectrally arbitrary tree sign pattern matrices. 
In this paper, we identify all 5 -by-5 tree sign pattern matrices with all edges negative. We get the following result from [16, Theorem 7.1].

LEMMA 4.1. There are no 5-by-5 spectrally arbitrary star sign pattern matrices with all edges negative.

If $A$ is a 5 -by-5 tree sign pattern matrix with all edges negative such that $G(A)$ is not a star, then $A$ is equivalent to one among

$$
P=\left[\begin{array}{ccccc}
p_{1} & + & 0 & 0 & 0 \\
- & p_{2} & + & 0 & 0 \\
0 & - & p_{3} & + & 0 \\
0 & 0 & - & p_{4} & + \\
0 & 0 & 0 & - & p_{5}
\end{array}\right], \quad R=\left[\begin{array}{ccccc}
r_{1} & + & + & + & 0 \\
- & r_{2} & 0 & 0 & 0 \\
- & 0 & r_{3} & 0 & 0 \\
- & 0 & 0 & r_{4} & + \\
0 & 0 & 0 & - & r_{5}
\end{array}\right],
$$

where $p_{1}, p_{2}, p_{3}, p_{4}, p_{5}, r_{1}, r_{2}, r_{3}, r_{4}, r_{5} \in\{+,-, 0\}$.

Let $n \geq 2$ and a sign pattern matrix of order $n$ be given by:

$$
T_{n}=\left[\begin{array}{cccccc}
- & + & 0 & \cdots & \cdots & 0 \\
- & 0 & + & \ddots & & \vdots \\
0 & - & 0 & + & \ddots & \vdots \\
\vdots & \ddots & \ddots & \ddots & \ddots & 0 \\
\vdots & & \ddots & - & 0 & + \\
0 & \cdots & \cdots & 0 & - & +
\end{array}\right] .
$$

Lemma 4.2 ([9]). Every super-pattern of $T_{n}$ is a spectrally arbitrary pattern for all $n \geq 2$.

If $G$ is a simple undirected graph, then the path $\left\{u=v_{0}, v_{1}, v_{2}, \ldots, v_{k-1}, v_{k}=v\right\}$ between two vertices $u$ and $v$ of $G$ has length $k$. The distance between $u$ and $v$, denoted by $d(u, v)$, is the length of the shortest path between them.

Lemma 4.3. Let $A$ be a path sign pattern matrix of order $n$ with all edges negative and $G(A)$ be the graph of $A$ with vertices $1,2, \ldots, n$ such that $i$ is adjacent to $i+1$ for $i=1,2, \ldots, n-1$. Suppose $A$ has a nonzero diagonal entry and $u_{\min }=\min \left\{u: a_{u u} \neq 0\right\}, u_{\max }=\max \left\{u: a_{u u} \neq 0\right\}$. If

$$
\max \left\{d\left(1, u_{\min }\right), d\left(n, u_{\max }\right)\right\} \geq\left\lfloor\frac{n}{2}\right\rfloor,
$$

then $A$ is not potentially nilpotent.

Proof. Let $C \in Q(A)$.

Let $n$ be even. If $d\left(1, u_{\min }\right) \geq \frac{n}{2}$, then $\left(C^{n}\right)_{11} \neq 0$. If $d\left(n, u_{\max }\right) \geq \frac{n}{2}$, then $\left(C^{n}\right)_{n n} \neq 0$. So $C$ is not nilpotent.

Let $n$ be odd. Suppose $d\left(1, u_{\min }\right) \geq \frac{n-1}{2}$. Let $w$ be the vertex of $G(A)$ such that $w<u_{\min }$ and $d\left(w, u_{\min }\right)=\frac{n-1}{2}$. Then $\left(C^{n}\right)_{w w} \neq 0$. So $C$ is not nilpotent. Similarly, if $d\left(n, u_{\max }\right) \geq \frac{n-1}{2}$, then $C$ is not nilpotent.

Hence, $A$ is not potentially nilpotent. 
577 Potentially stable and 5-by-5 spectrally arbitrary tree sign pattern matrices with all edges negative

LEMMA 4.4. The sign pattern matrices

$$
N_{1}=\left[\begin{array}{ccccc}
+ & + & 0 & 0 & 0 \\
- & 0 & + & 0 & 0 \\
0 & - & - & + & 0 \\
0 & 0 & - & - & + \\
0 & 0 & 0 & - & 0
\end{array}\right], N_{2}=\left[\begin{array}{ccccc}
+ & + & 0 & 0 & 0 \\
- & + & + & 0 & 0 \\
0 & - & - & + & 0 \\
0 & 0 & - & - & + \\
0 & 0 & 0 & - & 0
\end{array}\right], N_{3}=\left[\begin{array}{ccccc}
+ & + & 0 & 0 & 0 \\
- & - & + & 0 & 0 \\
0 & - & - & + & 0 \\
0 & 0 & - & - & + \\
0 & 0 & 0 & - & 0
\end{array}\right],
$$

do not allow a nilpotent matrix.

Proof. Let $b_{1}, b_{2}, b_{3}, b_{4}, a_{1}, a_{3}, a_{4}>0, a_{2} \in \mathbb{R}$ and

$$
C=\left[\begin{array}{ccccc}
a_{1} & 1 & 0 & 0 & 0 \\
-b_{1} & a_{2} & 1 & 0 & 0 \\
0 & -b_{2} & -a_{3} & 1 & 0 \\
0 & 0 & -b_{3} & -a_{4} & 1 \\
0 & 0 & 0 & -b_{4} & 0
\end{array}\right]
$$

The characteristic polynomial of $C$ is $z^{5}-c_{1} z^{4}+c_{2} z^{3}-c_{3} z^{2}+c_{4} z-c_{5}$, where

$$
\begin{gathered}
c_{1}=a_{1}+a_{2}-a_{3}-a_{4} ; \\
c_{2}=a_{1}\left(a_{2}-a_{3}-a_{4}\right)-a_{2}\left(a_{3}+a_{4}\right)+a_{3} a_{4}+b_{1}+b_{2}+b_{3}+b_{4} ; \\
c_{3}=a_{1}\left(b_{2}+b_{3}+b_{4}\right)+a_{2}\left(b_{3}+b_{4}\right)-a_{3}\left(b_{1}+b_{4}\right)-a_{4}\left(b_{1}+b_{2}\right)-a_{1} a_{2}\left(a_{3}+a_{4}\right)+\left(a_{1}+a_{2}\right) a_{3} a_{4} ; \\
c_{4}=b_{1} b_{3}+b_{1} b_{4}+b_{2} b_{4}+a_{1} a_{2}\left(b_{3}+b_{4}\right)-a_{1} a_{3} b_{4}-a_{1} a_{4} b_{2}-a_{2} a_{3} b_{4}+a_{3} a_{4} b_{1}+a_{1} a_{2} a_{3} a_{4} ; \\
c_{5}=a_{1} b_{2} b_{4}-a_{3} b_{1} b_{4}-a_{1} a_{2} a_{3} b_{4} .
\end{gathered}
$$

Suppose $C$ is nilpotent. Then, $c_{1}=c_{2}=c_{3}=c_{4}=c_{5}=0$.

Now $c_{1}=0 \Rightarrow a_{1}+a_{2}=a_{3}+a_{4}, c_{5}=0 \Rightarrow a_{1} b_{2}-a_{3} b_{1}-a_{1} a_{2} a_{3}=0$ and thus $b_{1}+a_{1} a_{2}>0$.

$$
\begin{aligned}
c_{3}=0 & \Rightarrow a_{1}\left(b_{3}+b_{4}\right)+a_{2}\left(b_{3}+b_{4}\right)-a_{3} b_{4}-a_{4}\left(b_{1}+b_{2}\right)-a_{1} a_{2} a_{4}+\left(a_{1}+a_{2}\right) a_{3} a_{4}=0 \\
& \Rightarrow\left(a_{1}+a_{2}\right) b_{3}+b_{4}\left(a_{1}+a_{2}-a_{3}\right)-a_{4}\left(b_{1}+b_{2}+a_{1} a_{2}-a_{1} a_{3}-a_{2} a_{3}\right)=0 \\
& \Rightarrow\left(a_{3}+a_{4}\right) b_{3}+a_{4} b_{4}-a_{4}\left(b_{1}+b_{2}+a_{1} a_{2}-a_{1} a_{3}-a_{2} a_{3}\right)=0 \\
& \Rightarrow b_{1}+b_{2}+a_{1} a_{2}-a_{1} a_{3}-a_{2} a_{3}>0 .
\end{aligned}
$$

Then $c_{4}=b_{1} b_{3}+b_{1} b_{4}+b_{2} b_{4}+a_{1} a_{2}\left(b_{3}+b_{4}\right)-a_{1} a_{3} b_{4}-a_{1} a_{4} b_{2}-a_{2} a_{3} b_{4}+a_{3} a_{4} b_{1}+a_{1} a_{2} a_{3} a_{4}$

$$
\begin{aligned}
& =b_{1} b_{3}+a_{1} a_{2} b_{3}+b_{4}\left(b_{1}+b_{2}+a_{1} a_{2}-a_{1} a_{3}-a_{2} a_{3}\right)+a_{4}\left(a_{3} b_{1}-a_{1} b_{2}+a_{1} a_{2} a_{3}\right) \\
& =\left(b_{1}+a_{1} a_{2}\right) b_{3}+b_{4}\left(b_{1}+b_{2}+a_{1} a_{2}-a_{1} a_{3}-a_{2} a_{3}\right)>0 .
\end{aligned}
$$

This is a contradiction. So $C$ is not nilpotent and thus none among $N_{1}, N_{2}, N_{3}$ allow a nilpotent matrix.

A sign pattern matrix $A$ is said to allow the inertia $\left(n_{1}, n_{2}, n_{3}\right)$ if there exists a $C \in Q(A)$ such that $i(C)=\left(n_{1}, n_{2}, n_{3}\right)$. 
Electronic Journal of Linear Algebra, ISSN 1081-3810

LEMMA 4.5. If the path sign pattern matrix $P$ in (4.1) is potentially nilpotent and allows the inertias $(5,0,0),(0,5,0)$, then $P$ is equivalent to some super-pattern of one among

$$
P_{1}=\left[\begin{array}{ccccc}
+ & + & 0 & 0 & 0 \\
- & 0 & + & 0 & 0 \\
0 & - & 0 & + & 0 \\
0 & 0 & - & 0 & + \\
0 & 0 & 0 & - & -
\end{array}\right], P_{2}=\left[\begin{array}{ccccc}
+ & + & 0 & 0 & 0 \\
- & 0 & + & 0 & 0 \\
0 & - & - & + & 0 \\
0 & 0 & - & 0 & + \\
0 & 0 & 0 & - & +
\end{array}\right], P_{3}=\left[\begin{array}{ccccc}
+ & + & 0 & 0 & 0 \\
- & 0 & + & 0 & 0 \\
0 & - & - & + & 0 \\
0 & 0 & - & + & + \\
0 & 0 & 0 & - & 0
\end{array}\right] .
$$

Proof. By Theorem 3.4,

$$
P=\left[\begin{array}{ccccc}
p_{1} & + & 0 & 0 & 0 \\
- & p_{2} & + & 0 & 0 \\
0 & - & p_{3} & + & 0 \\
0 & 0 & - & p_{4} & + \\
0 & 0 & 0 & - & p_{5}
\end{array}\right]
$$

allows the inertia $(0,5,0)$ if and only if at least one of $p_{1}, p_{3}, p_{5}$ is - . Similarly, $P$ allows the inertia $(5,0,0)$ if and only if at least one of $p_{1}, p_{3}, p_{5}$ is + . Hence, by Lemma 4.3 , the path sign pattern matrices of order 5 , which are potentially nilpotent and allow the inertias $(0,5,0)$ and $(5,0,0)$ can only be equivalent to some super-pattern of one among $P_{1}, P_{2}, P_{3}$ and

$$
P_{4}=\left[\begin{array}{ccccc}
+ & + & 0 & 0 & 0 \\
- & 0 & + & 0 & 0 \\
0 & - & - & + & 0 \\
0 & 0 & - & - & + \\
0 & 0 & 0 & - & 0
\end{array}\right]
$$

The only super-patterns of $P_{4}$ of the form $P$, which are super-patterns of none among $P_{1}, P_{2}, P_{3}$ are $N_{1}, N_{2}, N_{3}$. By Lemma 4.4, none among $N_{1}, N_{2}, N_{3}$ allow a nilpotent matrix. Hence, the result follows:

LEMMA 4.6. The sign pattern matrix

$$
\tilde{R}=\left[\begin{array}{ccccc}
r_{1} & + & + & + & 0 \\
- & r_{2} & 0 & 0 & 0 \\
- & 0 & r_{3} & 0 & 0 \\
- & 0 & 0 & 0 & + \\
0 & 0 & 0 & - & 0
\end{array}\right]
$$

is not potentially nilpotent, where $r_{1}, r_{2}, r_{3} \in\{+,-, 0\}$.

Proof. Let

$$
C=\left[\begin{array}{ccccc}
a_{1} & 1 & 1 & 1 & 0 \\
-b_{1} & a_{2} & 0 & 0 & 0 \\
-b_{2} & 0 & a_{3} & 0 & 0 \\
-b_{3} & 0 & 0 & 0 & 1 \\
0 & 0 & 0 & -b_{4} & 0
\end{array}\right] \in Q(\tilde{R})
$$

where $a_{1}, a_{2}, a_{3} \in \mathbb{R}$ and $b_{1}, b_{2}, b_{3}, b_{4}>0$. 
579 Potentially stable and 5-by-5 spectrally arbitrary tree sign pattern matrices with all edges negative

The characteristic polynomial of $C$ is $z^{5}-c_{1} z^{4}+c_{2} z^{3}-c_{3} x^{2}+c_{4} z-c_{5}$, where

$$
\begin{gathered}
c_{1}=a_{1}+a_{2}+a_{3}, \\
c_{2}=a_{2} a_{3}+a_{1} a_{2}+a_{1} a_{3}+b_{1}+b_{2}+b_{3}+b_{4}, \\
c_{3}=a_{1} b_{4}+a_{2}\left(b_{2}+b_{3}+b_{4}\right)+a_{3}\left(b_{1}+b_{3}+b_{4}\right)+a_{1} a_{2} a_{3}, \\
c_{4}=b_{4}\left(a_{2} a_{3}+a_{1} a_{2}+a_{1} a_{3}+b_{1}+b_{2}\right)+a_{2} a_{3} b_{3}, \\
c_{5}=b_{4}\left(a_{1} a_{2} a_{3}+a_{3} b_{1}+a_{2} b_{2}\right) .
\end{gathered}
$$

Suppose $C$ is a nilpotent matrix. Then $c_{1}=c_{2}=c_{3}=c_{4}=c_{5}=0$.

Case I: Assume $a_{1} \neq 0$.

Then, $c_{1}=0 \Rightarrow a_{1}+a_{2}+a_{3}=0$ and $c_{5}=0 \Rightarrow a_{1} a_{2} a_{3}+a_{3} b_{1}+a_{2} b_{2}=0$.

Hence, $c_{3}=0 \Rightarrow a_{2}+a_{3}=0 \Rightarrow a_{1}=0$, a contradiction.

Case II: Assume $a_{1}=0$.

Then, $c_{2}=0 \Rightarrow b_{1}+b_{2}+a_{2} a_{3}<0$ and $c_{1}=0 \Rightarrow a_{2}+a_{3}=0 \Rightarrow a_{2} a_{3} \leq 0$.

Therefore, $c_{4}=b_{4}\left(a_{2} a_{3}+b_{1}+b_{2}\right)+a_{2} a_{3} b_{3}<0$, a contradiction.

Hence, $C$ is not a nilpotent matrix and thus $\tilde{R}$ is not potentially nilpotent.

LEMMA 4.7. The sign pattern matrix $R$ in (4.1) is spectrally arbitrary if and only if it is equivalent to some super-pattern of one among

$$
R_{1}=\left[\begin{array}{ccccc}
0 & + & + & + & 0 \\
- & + & 0 & 0 & 0 \\
- & 0 & - & 0 & 0 \\
- & 0 & 0 & - & + \\
0 & 0 & 0 & - & 0
\end{array}\right], R_{2}=\left[\begin{array}{ccccc}
0 & + & + & + & 0 \\
- & + & 0 & 0 & 0 \\
- & 0 & - & 0 & 0 \\
- & 0 & 0 & 0 & + \\
0 & 0 & 0 & - & -
\end{array}\right], R_{3}=\left[\begin{array}{ccccc}
0 & + & + & + & 0 \\
- & + & 0 & 0 & 0 \\
- & 0 & 0 & 0 & 0 \\
- & 0 & 0 & - & + \\
0 & 0 & 0 & - & +
\end{array}\right] .
$$

Proof. We have

$$
R=\left[\begin{array}{ccccc}
r_{1} & + & + & + & 0 \\
- & r_{2} & 0 & 0 & 0 \\
- & 0 & r_{3} & 0 & 0 \\
- & 0 & 0 & r_{4} & + \\
0 & 0 & 0 & - & r_{5}
\end{array}\right]
$$

Suppose $R$ is spectrally arbitrary. Then, $R$ is potentially nilpotent and allows the inertias $(5,0,0)$ and $(0,5,0)$. Since $R$ allows the inertia $(0,5,0)$, from Case II in the proof of Theorem $3.7, R$ is permutation similar to some super-pattern of one among

$$
A_{1}=\left[\begin{array}{ccccc}
0 & + & + & + & 0 \\
- & - & 0 & 0 & 0 \\
- & 0 & 0 & 0 & 0 \\
- & 0 & 0 & 0 & + \\
0 & 0 & 0 & - & 0
\end{array}\right], A_{2}=\left[\begin{array}{ccccc}
0 & + & + & + & 0 \\
- & + & 0 & 0 & 0 \\
- & 0 & 0 & 0 & 0 \\
- & 0 & 0 & - & + \\
0 & 0 & 0 & - & +
\end{array}\right]
$$


Now

$$
S(-R) S=\left[\begin{array}{ccccc}
-r_{1} & + & + & + & 0 \\
- & -r_{2} & 0 & 0 & 0 \\
- & 0 & -r_{3} & 0 & 0 \\
- & 0 & 0 & -r_{4} & + \\
0 & 0 & 0 & - & -r_{5}
\end{array}\right], \quad \text { where } S=\left[\begin{array}{ccccc}
+ & 0 & 0 & 0 & 0 \\
0 & - & 0 & 0 & 0 \\
0 & 0 & - & 0 & 0 \\
0 & 0 & 0 & - & 0 \\
0 & 0 & 0 & 0 & +
\end{array}\right] .
$$

So both $A_{2}$ and $S\left(-A_{2}\right) S$ allow the inertia $(0,5,0)$. Since the inertia of a matrix remains unchanged after a similarity transformation, both $A_{2}$ and $-A_{2}$ allow the inertia $(0,5,0)$, that is, $A_{2}$ allows the inertias $(0,5,0)$ and $(5,0,0)$. If $R$ allows the inertia $(0,5,0)$ and $R$ is not permutation similar to any super-pattern of $A_{2}$, then $R$ must be permutation similar to some super-pattern of $A_{1}$. Any super-pattern of $A_{1}$, which is not equivalent to any super-pattern of $A_{2}$ and allows the inertia $(5,0,0)$, must be equivalent to some super-pattern of

$$
A_{3}=\left[\begin{array}{ccccc}
0 & + & + & + & 0 \\
- & + & 0 & 0 & 0 \\
- & 0 & - & 0 & 0 \\
- & 0 & 0 & 0 & + \\
0 & 0 & 0 & - & 0
\end{array}\right]
$$

Therefore, if $R$ is spectrally arbitrary, then by Lemma 4.6, we can conclude that $R$ must be equivalent to some super-pattern of one among $R_{1}, R_{2}, R_{3}$.

For the converse part, let $X=\left[\begin{array}{ccccc}0 & 1 & 1 & 1 & 0 \\ -x_{0} & x_{1} & 0 & 0 & 0 \\ -x_{2} & 0 & 0 & 0 & 0 \\ -x_{3} & 0 & 0 & -x_{4} & 1 \\ 0 & 0 & 0 & -x_{5} & x_{6}\end{array}\right] \in Q\left(R_{3}\right)$, where $x_{0}, x_{1}, x_{2}, x_{3}, x_{4}, x_{5}, x_{6}>0$.

The characteristic polynomial of $X$ is $z^{5}-c_{1} z^{4}+c_{2} z^{3}-c_{3} z^{2}+c_{4} z-c_{5}$, where

$$
\begin{gathered}
c_{1}=x_{1}-x_{4}+x_{6}, \\
c_{2}=x_{0}+x_{2}+x_{3}+x_{5}-x_{1} x_{4}+x_{1} x_{6}-x_{4} x_{6}, \\
c_{3}=x_{1} x_{2}+x_{1} x_{3}+x_{1} x_{5}-x_{0} x_{4}-x_{2} x_{4}+x_{0} x_{6}+x_{2} x_{6}+x_{3} x_{6}-x_{1} x_{4} x_{6}, \\
c_{4}=\left(x_{0}+x_{2}\right)\left(x_{5}-x_{4} x_{6}\right)-x_{1} x_{2} x_{4}+x_{1} x_{6}\left(x_{2}+x_{3}\right), \\
c_{5}=x_{1} x_{2}\left(x_{5}-x_{4} x_{6}\right) .
\end{gathered}
$$

$X$ has a nilpotent realization at $\left(\hat{x}_{0}, \hat{x}_{1}, \hat{x}_{2}, \hat{x}_{3}, \hat{x}_{4}, \hat{x}_{5}, \hat{x}_{6}\right)=(2,2,1,1,4,8,2)$. The Jacobian of the coefficients of $z^{4}, z^{3}, z^{2}, z^{1}, z^{0}$ with respect to $x_{0}, x_{1}, x_{2}, x_{3}, x_{5}$ at $(2,2,1,1,4,8,2)$ is

$$
\begin{aligned}
& \left|\begin{array}{ccccc}
0 & -1 & 0 & 0 & 0 \\
1 & -x_{4}+x_{6} & 1 & 1 & 1 \\
x_{4}-x_{6} & -x_{2}-x_{3}-x_{5}+x_{4} x_{6} & -x_{1}+x_{4}-x_{6} & -x_{1}-x_{6} & -x_{1} \\
x_{5}-x_{4} x_{6} & -x_{2} x_{4}+x_{6}\left(x_{2}+x_{3}\right) & x_{5}-x_{4} x_{6}-x_{1} x_{4}+x_{1} x_{6} & x_{1} x_{6} & x_{0}+x_{2} \\
0 & -x_{2}\left(x_{5}-x_{4} x_{6}\right) & -x_{1}\left(x_{5}-x_{4} x_{6}\right) & 0 & -x_{1} x_{2}
\end{array}\right| \\
& =\left|\begin{array}{ccccc}
0 & -1 & 0 & 0 & 0 \\
1 & -2 & 1 & 1 & 1 \\
2 & -2 & 0 & -4 & -2 \\
0 & 0 & -4 & 4 & 3 \\
0 & 0 & 0 & 0 & -2
\end{array}\right|=-2\left|\begin{array}{ccc}
1 & 1 & 1 \\
2 & 0 & -4 \\
0 & -4 & 4
\end{array}\right|=64 \neq 0 .
\end{aligned}
$$


581 Potentially stable and 5-by-5 spectrally arbitrary tree sign pattern matrices with all edges negative

Therefore, by the Nilpotent-Jacobian method, every super-pattern of $R_{3}$ is spectrally arbitrary. Similarly, the super-patterns of the sign pattern matrices $R_{1}$ and $R_{2}$ can be shown to be spectrally arbitrary by the Nilpotent-Jacobian method. Such nilpotent matrices in $Q\left(R_{1}\right)$ and $Q\left(R_{2}\right)$ are respectively,

$$
\left[\begin{array}{ccccc}
0 & 1 & 1 & 1 & 0 \\
-\frac{9+5 \sqrt{3}}{3} & 2+\sqrt{3} & 0 & 0 & 0 \\
-\frac{3+\sqrt{3}}{3} & 0 & -1 & 0 & 0 \\
-\frac{2+\sqrt{3}}{2} & 0 & 0 & -1-\sqrt{3} & 1 \\
0 & 0 & 0 & -\frac{2+\sqrt{3}}{2} & 0
\end{array}\right],\left[\begin{array}{ccccc}
0 & 1 & 1 & 1 & 0 \\
-\frac{9+5 \sqrt{3}}{6} & \sqrt{3}+1 & 0 & 0 & 0 \\
-\frac{9-5 \sqrt{3}}{6} & 0 & -\sqrt{3}+1 & 0 & 0 \\
-1 & 0 & 0 & 0 & 1 \\
0 & 0 & 0 & -2 & -2
\end{array}\right] .
$$

Since any sign pattern matrix equivalent to a spectrally arbitrary pattern is also spectrally arbitrary, the converse part follows.

The following result characterizes all 5-by-5 spectrally arbitrary tree sign pattern matrices with all edges negative.

THEOREM 4.8. A 5-by-5 tree sign pattern matrix with all edges negative is spectrally arbitrary if and only if it is equivalent to some super-pattern of one among $P_{1}, P_{2}, P_{3}, R_{1}, R_{2}, R_{3}$.

Proof. Let $A$ be a 5 -by-5 spectrally arbitrary tree sign pattern matrix with all edges negative. Then by Lemma 4.1, $A$ is not a star sign pattern matrix. If $A$ is a path sign pattern matrix, then it is equivalent to a matrix of the form $P$ in (4.1). If $A$ is spectrally arbitrary, then $A$ is potentially nilpotent and allows the inertias $(5,0,0)$ and $(0,5,0)$. So by Lemma $4.5, A$ is equivalent to some super-pattern of one among $P_{1}, P_{2}, P_{3}$.

If $A$ is neither a star sign pattern matrix nor a path sign pattern matrix, then it is equivalent to a matrix of the form $R$ in (4.1). If $A$ is spectrally arbitrary, then by Lemma 4.7, $A$ must be equivalent to some super-pattern of one among $R_{1}, R_{2}, R_{3}$.

Now we prove the converse part. By Lemma 4.2, the super-patterns of $P_{1}$ are spectrally arbitrary. The super-patterns of the sign pattern matrices $P_{2}$ and $P_{3}$ can be shown to be spectrally arbitrary by the Nilpotent-Jacobian method, and such nilpotent matrices in $Q\left(P_{2}\right)$ and $Q\left(P_{3}\right)$ are respectively,

$$
\left[\begin{array}{ccccc}
\sqrt{5}+1 & 1 & 0 & 0 & 0 \\
-4 & 0 & 1 & 0 & 0 \\
0 & -4 & -2 \sqrt{5} & 1 & 0 \\
0 & 0 & -4 & 0 & 1 \\
0 & 0 & 0 & -4 & \sqrt{5}-1
\end{array}\right],\left[\begin{array}{ccccc}
6 & 1 & 0 & 0 & 0 \\
-12 & 0 & 1 & 0 & 0 \\
0 & -24 & -12 & 1 & 0 \\
0 & 0 & -54 & 6 & 1 \\
0 & 0 & 0 & -18 & 0
\end{array}\right]
$$

The super-patterns of the sign pattern matrices $R_{1}, R_{2}$, and $R_{3}$ are spectrally arbitrary by Lemma 4.7. Since any sign pattern matrix equivalent to a spectrally arbitrary pattern is also spectrally arbitrary, the converse part follows.

Acknowledgment. The author is grateful to Indian Institute of Technology Guwahati, India, for providing him with a graduate fellowship to carry out research. The author is grateful to Dr. Sriparna Bandyopadhyay for careful reading and suggestions for a better presentation of the paper. The author is also grateful to the reviewer(s) for their comments and suggestions which help to improve the paper and thankful to the Editor-in-Chief for giving the author a chance to revise. 


\section{REFERENCES}

[1] M. Arav, F. Hall, Z. Li, K. Kaphle, and N. Manzagol. Spectrally arbitrary tree sign patterns of order 4. Electron. J. Linear Algebra, 20:180-197, 2010.

[2] T. Bone. Positive feedback may sometimes promote stability. Linear Algebra Appl., 51:143-151, 1983.

[3] T. Britz, J.J. McDonald, D.D. Olesky, and P. van den Driessche. Minimal spectrally arbitrary sign patterns. SIAM J. Matrix Anal. Appl., 26(1):257-271, 2004.

[4] J. Cavalcanti. Potentially Hurwitz structures: A characterization of nests. In: 2020 59th IEEE Conference on Decision and Control (CDC), 5180-5187, 2020.

[5] M. Cavers. Polynomial stability and potentially stable patterns Linear Algebra Appl., 613:87-114, 2021.

[6] J.H. Drew, C.R. Johnson, D.D. Olesky, and P. van den Driessche. Spectrally arbitrary patterns. Linear Algebra Appl., 308(1-3):121-137, 2000.

[7] Y. Gao and J. Li. On the potential stability of star sign pattern matrices. Linear Algebra Appl., 327(1-3):61-68, 2001.

[8] D.A. Grundy, D.D. Olesky, and P. van den Driessche. Constructions for potentially stable sign patterns. Linear Algebra Appl., 436(12):4473-4488, 2012.

[9] C. Garnett and B.L. Shader. A proof of the $T_{n}$ conjecture: centralizers, Jacobians and spectrally arbitrary sign patterns. Linear Algebra Appl., 436(12):4451-4458, 2012.

[10] J.M. Harris, J.L. Hirst, and M.J. Mossinghoff. Combinatorics and Graph Theory, Second edition. Undergraduate Texts in Mathematics. Springer, New York, 2008.

[11] R.A. Horn and C.R. Johnson. Matrix Analysis, Second edition. Cambridge University Press, Cambridge, 2013.

[12] F.J. Hall and Z. Li. In: L. Hogben (editor), Handbook of Linear Algebra, Second edition. Discrete Mathematics and Its Applications. CRC Press, Boca Raton, FL, 2014.

[13] C. Jeffries and C.R. Johnson. Some sign patterns that preclude matrix stability. SIAM J. Matrix Anal. Appl., 9(1):19-25, 1988.

[14] C.R. Johnson, J.S. Maybee, D.D. Olesky, and P. van den Driessche. Nested sequences of principal minors and potential stability. Linear Algebra Appl., 262:243-257, 1997.

[15] C.R. Johnson and T.A. Summers. The potentially stable tree sign patterns for dimensions less than five. Linear Algebra Appl., 126:1-13, 1989.

[16] G. MacGillivray, R.M. Tifenbach, and P. van den Driessche. Spectrally arbitrary star sign patterns. Linear Algebra Appl., 400:99-119, 2005.

[17] D.D. Olesky, M.J. Tsatsomeros, and P. van den Driessche. Sign patterns with a nest of positive principal minors. Linear Algebra Appl., 436(12):4392-4399, 2012. 